\title{
Physiological and behavioral differences in sensory processing: a comparison of children with Autism Spectrum Disorder and Sensory Modulation Disorder
}

\author{
Sarah A. Schoen ${ }^{1,2,3 *}$, Lucy J. Miller ${ }^{1,2,3}$, Barbara A. Brett-Green ${ }^{1,2}$ and Darci M. Nielsen \\ Sensory Processing Disorder Foundation, Greenwood Village, CO, USA \\ 2 University of Colorado at Denver, Denver, CO, USA \\ ${ }^{3}$ Rocky Mountain University of Health Professionals, Provo, UT, USA
}

Edited by:

Barry E. Stein, Wake Forest University, USA

\section{Reviewed by:}

Sophie Molholm, The City College of New York, USA

Mark Wallace, Vanderbilt University, USA

\section{*Correspondence:}

Sarah A. Schoen, Director of Applied

Research, Sensory Processing

Disorder Foundation, 5655 South

Yosemite Street, Suite 304,

Greenwood Village, CO 80111, USA

e-mail: schoen@SPDfoundation.net
A high incidence of sensory processing difficulties exists in children with Autism Spectrum Disorder (ASD) and children with Sensory Modulation Disorder (SMD). This is the first study to directly compare and contrast these clinical disorders. Sympathetic nervous system markers of arousal and reactivity were utilized in a laboratory paradigm that administered a series of sensory challenges across five sensory domains. The Short Sensory Profile, a standardized parent-report measure, provided a measure of sensory-related behaviors. Physiological arousal and sensory reactivity were lower in children with ASD whereas reactivity after each sensory stimulus was higher in SMD, particularly to the first stimulus in each sensory domain. Both clinical groups had significantly more sensory-related behaviors than typically developing children, with contrasting profiles. The ASD group had more taste/smell sensitivity and sensory under-responsivity while the SMD group had more atypical sensory seeking behavior. This study provides preliminary evidence distinguishing sympathetic nervous system functions and sensory-related behaviors in Autism Spectrum Disorder and Sensory Modulation Disorder. Differentiating the physiology and sensory symptoms in clinical groups is essential to the provision of appropriate interventions.

Keywords: sensory processing, arousal, reactivity, sensory modulation, Autism Spectrum Disorder

\section{INTRODUCTION}

Sensory processing involves the ability to take in, organize and make sense of different kinds of sensations received by the brain. Rates of sensory processing dysfunction may be as high as $90 \%$ in individuals with Autism Spectrum Disorder (ASD) (Baranek et al., 2006; Leekam et al., 2007; Tomchek and Dunn, 2007; Baker et al., 2008) and are estimated to be between $5 \%$ and $16 \%$ in the general population (Ahn et al., 2004; Ben-Sasson et al., 2009). When children present with sensory processing impairments in the absence of any other childhood disorder it is known as idiopathic Sensory Processing Disorder (SPD) (Miller et al., 2007a). There are three primary subtypes of SPD: Sensory Modulation Disorder, Sensory-based Motor Disorder and Sensory Discrimination Disorder (Miller et al., 2007a). The focus of this paper is on Sensory Modulation Disorder, which is characterized by difficulty regulating one's responses (i.e. duration, intensity, and/or type of response) in a flexible and adaptive manner to sensory experiences that occur in daily life. Because SMD is related to grading one's responses to sensations from the environment, patterns of responsivity may vary throughout the day and from day to day depending on the context (World Health Organization, 2001; Zero To Three, 2005; Miller et al., 2007a). Therefore, to be considered a disorder, the responses to sensory input must significantly impair the successful performance of one's daily activities and routines.

The literature on sensory processing disorders primarily utilizes two forms of data: (1) parent/caregiver report measures that describe sensory-related behaviors and (2) physiological measures that provide information about arousal and sensory reactivity. Atypical behavioral and physiologic responses to sensory stimuli are reported in various groups with clinical diagnoses (Ermer and Dunn, 1998; Miller et al., 1999; Rogers et al., 2003; Leekam et al., 2007), but few studies compare sensory symptoms across clinical conditions. Both individuals with ASD as well as children with idiopathic SMD present with behavioral and physiological features attributed to sensory processing difficulties that are qualitatively and quantitatively different from typically developing children (Kientz and Dunn, 1997; Baranek, 1999; McIntosh et al., 1999a; Watling et al., 2001; Leekam et al., 2007; Tomchek and Dunn, 2007; Kern et al., 2008; Reynolds and Lane, 2008). Clinical evidence suggests that the sensory-related behaviors in ASD and SMD may overlap, but no studies directly compare the sensory processing dysfunction of these groups (Baranek, 1999; Watling et al., 2001; Rogers et al., 2003; Leekam et al., 2007). A better understanding of the similarities and differences in sensory functioning between these two clinical disorders is crucial to differential diagnosis and can have a profound impact on treatment planning (Baranek et al., 2007).

In both ASD and SMD, the atypical sensory processing is associated with significant problems in adaptive behavior and participation in daily life activities (Cohn et al., 2000; Rogers et al., 2003; Kern et al., 2006; Hilton et al., 2007; Bar-Shalita et al., 2008). Children with sensory processing difficulties often suffer from impaired selfesteem, anxiety, depression, or aggression (Pfeiffer et al., 2005), that result in problems in social participation (Baker et al., 2008), 
self-regulation, and impaired sensorimotor skills needed for daily life tasks (Cohn et al., 2000; Talay-Ongan and Wood, 2000; Smith et al., 2005; Ashburner et al., 2008). Lack of participation in daily sensory experiences (e.g. avoidance of playground activities, play with textured materials, or social interactions) can also significantly impact a child's learning opportunities due to decreased active exploration of the environment (Baranek, 2002).

\section{SENSORY PROCESSING IN CHILDREN WITH ASD}

A wide range of sensory disturbances are reported in children with ASD (Baranek, 1999; Iarocci and McDonald, 2006; Liss et al., 2006; Ben-Sasson et al., 2007; Kern et al., 2007; Baker et al., 2008; Minshew and Hobson, 2008). Evidence suggests the profile of atypical sensory-related behaviors in children with ASD is different than children with other developmental disorders (Ermer and Dunn, 1998; Rogers et al., 2003; Baranek et al., 2006), but studies differ on the sensory domains highlighted. For example, a study of sensory symptoms in ASD compared to children with mental retardation noted greater tactile seeking (rubbing objects, flicking fingers), movement seeking (rocking, jumping), visual avoidance (poor eye contact) and auditory under-responsivity (lack of response to verbal input) in children with ASD (Adrien et al., 1987). Rogers et al. (2003) found more sensory symptoms in children with ASD compared to children with developmental disabilities of mixed etiology, but no difference compared to children with Fragile X syndrome. In their study, children with ASD scored most impaired in taste/smell sensitivity on the Short Sensory Profile (McIntosh et al., 1999b) compared to all other groups (Rogers et al., 2003). Similarly, Leekam et al. (2007) found a greater number of sensory symptoms in children with ASD compared to children with developmental disability or language impairment, especially in the taste/smell domain. Ermer and Dunn (1998) conducted a discriminant analysis identifying factors on the Sensory Profile (Dunn, 1997b) that differentiated children with ASD from children with Attention Deficit/Hyperactivity Disorder (ADHD). They found that children with ASD had a lower incidence of sensory seeking behaviors and a higher incidence of oral sensitivity. Using the Sensory Experiences Questionnaire, Baranek et al. (2006) suggests the feature that most discriminates preschool children with autism from typically developing peers, as well as from developmentally delayed children is under-responsiveness to both nonsocial (i.e. objects and materials) and social stimuli. However, none of these studies compared children with ASD to children with idiopathic SMD.

\section{SENSORY MODULATION DISORDER}

One subtype of Sensory Processing Disorder is called Sensory Modulation Disorder (SMD). It is characterized by difficulty responding to sensory input in a flexible and adaptive manner in order to participate successfully in daily life. Children with SMD can display a range of sensory symptoms. One nosology of SMD includes three subtypes, Sensory Over-responsivity, Sensory Underresponsivity and Sensory Seeking/Craving (Miller et al., 2007b). Individuals with Sensory Over-responsivity often display negative responses to touch, sound or bright lights. Behaviorally they avoid certain sensory experiences or have extreme emotional reactions to typically non-aversive sensory stimuli (Kinnealey et al., 1995;
Dunn, 1997a; Bar-Shalita et al., 2005; Miller et al., 2007b). On the other hand, individuals with Sensory Under-responsivity ignore or do not notice typical sensory stimuli (Dunn, 2001; Miller et al., 2007b). They appear passive, uninterested in the environment and often lazy or lethargic, which is hypothesized to be caused by the inability of the sensory information to reach their threshold for awareness. Lastly, individuals with Sensory Seeking/Craving excessively crave sensory experiences (Miller et al., 2007b) and are described behaviorally as "always on the go", often appearing reckless and dangerous in their attempts to fulfill their sensory needs. There is also a wide range of normal responses to sensory input. Therefore, individuals are only considered to have a "disorder" when their responses to sensory input are so extreme that it interferes with daily functioning at home (i.e. self-care, eating, sleeping), in school and in interactions with peers or adults.

\section{PHYSIOLOGICAL MEASURES}

Preliminary physiological evidence implicates both sympathetic (McIntosh et al., 1999b; Schoen et al., 2008a) and parasympathetic impairments (Schaaf et al., 2003) in individuals with atypical sensory processing (McIntosh et al., 1999b; Schaaf et al., 2003; Schoen et al., 2008a). McIntosh et al. (1999b) reported increased sympathetic reactivity and slower habituation as measured by electrodermal activity (EDA) in children with idiopathic SMD and Schaaf et al. (2003) found decreased parasympathetic activity measured by Vagal tone. Common to these studies was the use of a laboratory paradigm called the Sensory Challenge Protocol Space Lab. This paradigm measures physiological arousal and reactivity of participants to a series of challenges across five sensory domains: auditory, visual, tactile, olfactory and movement (Miller et al., 1999, 2001). However, these pilot studies have not yet been replicated. Additionally, these earlier studies include only reactivity to sensory stimuli and did not examine physiological arousal prior to or following the sensory challenges.

While EDA is useful for indexing psychological processes (e.g. anxiety), it may be more difficult to identify specific brain centers and pathways given its multiple levels of control. EDA is influenced by the reticular formation (Sequeira et al., 2009), the hypothalamus, limbic system (i.e. amygdala, hippocampus, and cingulate gyrus) and frontal cortex (Lee et al., 1988; Mangina and BeuzeronMangina, 1996; Sequeira et al., 2009). EDA is often used as a general arousal/attention indicator. For example, change in skin conductance level in the absence of a stimulus is an important indicator of an individual's state of arousal and alertness. During the Sensory Challenge Protocol, skin conductance responses are hypothesized to be the result of "perceived" significance of the sensory stimulus as well as reflecting affective processes in response to the "perceived" impact of the stimulus. EDA changes associated with orienting and attention are likely modulated by prefrontal cortical activity; and EDA changes associated with affect are likely modulated by the amygdala and limbic system (Edelberg, 1973; Boucsein, 1992; Hugdahl, 1995; Dawson et al., 2000).

Findings from research examining arousal and reactivity of children with ASD using physiological markers of sympathetic nervous system functioning have produced differing results. In general, two patterns have been reported: (1) a high degree of non-responding (van Engeland et al., 1991) and (2) high resting (baseline) levels 
of arousal (van Engeland et al., 1991; Hirstein et al., 2001). Less consistent findings are reported for reactivity measures, with both higher reactivity (James and Barry, 1984; Barry and James, 1988; Hirstein et al., 2001) as well as no differences compared to typical controls (Palkovitz and Wiesenfeld, 1980; Stevens and Gruzelier, 1984). It is suggested that individuals with ASD may have two different patterns of responding, (1) a pattern of high arousal and high reactivity or (2) a pattern of low arousal and low reactivity (Hirstein et al., 2001). However, the lack of a typical comparison group precluded determining if these patterns were different from typically developing children. The sensory abnormalities in ASD were hypothesized to be related to over-arousal (Hutt and Hutt, 1964; Dawson and Lewy, 1989) and under-arousal (Rimland, 1964; DesLauriers and Carlson, 1969), but empirical evidence is scarce related to the underlying physiology (Rogers and Ozonoff, 2005).

Clinical evidence suggests that the sensory symptoms in ASD and SMD overlap and differential diagnosis of these two groups may be confounded by similarities in sensory-related abnormalities. To date, no studies have compared the behavioral and physiological profiles of children with ASD to those who have idiopathic SMD. Studies comparing sympathetic markers of arousal and reactivity to sensory stimuli and behavioral symptoms are needed. Describing different behavioral features of atypical sensory processing along with an examination of physiological measures may be crucial in differentiating clinical groups. Understanding the sensory problems of both ASD and SMD is essential to the provision of appropriate treatments.

The purpose of this study was to evaluate physiological and behavioral measures of children with ASD and SMD and to address the following questions:

1. Do children with ASD have different markers of sympathetic nervous system functioning, as measured by electrodermal activity (EDA), during sensory challenges compared to children with SMD?

2. Do children with ASD have different sensory-related behaviors, as measured by the Short Sensory Profile, compared to children with SMD?

It is hypothesized that children with SMD have atypical physiological reactivity, while children with ASD have atypical arousal (Schoen et al., 2008b). In addition, differences in sensory-related behaviors are hypothesized in sensory under-responsivity and taste/ smell sensitivity (Miller et al., 2001; Rogers et al., 2003; Ben-Sasson et al., 2009).

\section{MATERIALS AND METHODS PARTICIPANTS}

Forty children with Autism Spectrum Disorder (i.e. Asperger Syndrome or High Functioning Autism) ages 5-15 (mean = 9.3, $\mathrm{SD}=2.74$ ) participated in this study. Participants were recruited from the Autism and Developmental Disabilities Research Group of the University of Colorado, Denver, where they were evaluated by licensed clinical psychologists with specialized training in assessment of ASD. Children met stringent diagnostic criteria for ASD based on clinical judgment and scores above the cutoff on the Autism Diagnostic Observation Scale (ADOS, Lord et al.,
1999) and the Social Communication Questionnaire (Berument et al., 1999). Full-scale IQ scores were above 70 on the LeiterRevised International Performance Scale, a nonverbal IQ scale $(M=100 ; \mathrm{SD}=15)$. A diagnosis of either Asperger Syndrome or High Functioning Autism was determined by the experienced psychologists, relying upon the Autism Diagnostic Observation Scale, the Social Communication Questionnaire, and developmental history data.

Of the 40 children referred for the study, two were unable to complete testing due to anticipation anxiety related to the laboratory protocol. Thus, the sample size for the study was 38, with 11 Asperger Syndrome and 27 High Functioning Autism participants. Ninety-three percent of the ASD participants were male and $80 \%$ were Caucasian.

Thirty-one children with Sensory Modulation Disorder (SMD), ages $5-13$ years $(M=8.0, \mathrm{SD}=1.93)$ participated in this study. Participants were recruited from the Sensory Therapies And Research (STAR) Center, a multi-disciplinary private clinic near Denver, CO. All children were referred for atypical sensory responsivity by a clinician, pediatrician, psychologist or parent. Inclusion was based on a comprehensive Occupational Therapy Assessment by certified occupational therapists with specialized training and mentorship in identifying SMD. A combination of clinical observation and in-depth parent interview of sensory functioning related to SMD were used to assess children. Clinical observations included responses to sensory experiences during a standardized developmental motor scale, clinical observations in an occupational therapy gym of responses to sensory activities and materials. Specific guidelines for identifying SMD are available in two recently published diagnostic manuals: DC-0-3 (Zero To Three, 2005) and the Diagnostic Manual for Infancy and Early Childhood (2005). Appendix III of the Diagnostic Manual for Infancy and Early Childhood (2005) served as a guide for the clinical assessment of SMD in this study and provided the structure for both the observations of the child and the caregiver/parent questions and interview concerning aspects of sensory functioning. For example: a child who avoids exploring the environment and appears to display overt signs of "fight or flight" behaviors when presented with particular sensory stimuli reflects sensory over-responsivity; a child who does not attend or orient to salient stimuli in the environment would indicate sensory under-responsivity; a child who craves high intensity sensory input, tends to get over-aroused and behaviorally disorganized is suggestive of sensory seeking/craving. A more complete description and sample questions are available in Appendix III, on pages 289-305 (Diagnostic Manual for Infancy and Early Childhood 2005).

Currently there is no reliability and validity data on this method of SMD assessment. Therefore, all observations using this guide are considered clinical. The final determination that a child had SMD was a global/overall impression of the occupational therapist based on the available evidence (i.e. child observation and parent interview). Children with medical conditions such as cerebral palsy, Down syndrome, fragile $\mathrm{X}$ syndrome and those who had global developmental delays were excluded. Four of the participants had clinical diagnosis of Attention deficit/hyperactivity disorder, one of whom also had a diagnosis of generalized anxiety disorder. The SMD study participants were $77 \%$ male and $87 \%$ Caucasian. 
Thirty-three typically developing children (TYP) ranging in age from $4-12($ mean $=8.1, \mathrm{SD}=2.44)$ were recruited from the University of Colorado at Denver. All participants passed a Telephone Screen for Recruitment ensuring that they did not have any of the following: birth risk factors, history of neurological abnormalities, behavioral or learning disabilities and/or unusual sensory sensitivities. Parents of typically developing children reported age appropriate behavior and learning abilities. Forty-seven percent of the typical participants were male and $75 \%$ were Caucasian.

Intelligence IQ was not assessed for the typical or SMD groups. Based on previous experience, lower IQs are not expected in children with SMD or typical controls (McIntosh et al., 1999b). IQ was assessed for the ASD group because of the wide variability in this population and the report of a relationship between IQ and physiology (Stevens and Gruzelier, 1984; Martinez-Selva et al., 1995). Participants over 7 years and all parents provided written consent, using procedures approved by the Institutional Review Board of the University of Colorado, Denver.

\section{INSTRUMENTATION}

\section{Physiologic laboratory protocol}

The Sensory Challenge Protocol Space Lab is a physiologic laboratory paradigm (McIntosh et al., 1999b; Miller et al., 1999, 2001; Mangeot et al., 2001; Hagerman et al., 2002) during which arousal (tonic) and reactivity (phasic) measures of electrodermal activity (EDA) are collected with palmar electrodes using the PSYLAB System (Contact Precision Instruments, Cambridge MA).

The laboratory is decorated to look like a pretend spaceship with walls painted to look like three dimensional space ship panels and with low light levels in the room. A small console, the "control panel for our space ship", is centered in front of the child with video monitor and strobe light. The child is seated in a sturdy armchair mounted on a motorized tilting frame. As the experimenter attaches electrodes to the child, the child watches an appropriate part of Apollo 13, depicting astronauts donning spacesuits and being strapped into the spaceship. Subsequently, EDA is recorded continuously during a 3-min baseline period, followed by the presentation of 48 sensory stimuli (Miller et al., 1999, 2001). Eight trials are administered to the participant in the following order during the sensory phases of the paradigm: auditory (tone), visual (flash), auditory (siren), olfactory (wintergreen), tactile (feather) and vestibular (chair tip). Each 3 -s stimulus is presented in a pseudo-random schedule 10-15 s apart. Participants are not specifically directed to attend to the stimuli nor are they required to complete a task. Presentations of auditory, visual, and vestibular stimuli are automatically controlled by the Psylab computer program. Olfactory and tactile stimuli are administered by a trained experimenter, who receives instructions through headphones so that administration of the stimuli is consistent and synchronized with the computer program. The computer program automatically tags the onset of each of stimuli in the EDA data record so that skin conductance responses to the discrete stimulus can be analyzed. Discrete stimulus elicited skin conductance responses are only recorded if the onset is between 0.8 and $5 \mathrm{~s}$ after the stimulus and the response is at least 0.02 microSeimans $(\mu \mathrm{S})$. The signals are sampled at $1000 \mathrm{~Hz}$, digitized, stored on a computer, and later reduced using PSYLAB (Contact Precision Instruments, Cambridge MA). The experiment ends with a 3-min recovery period during which no stimuli are delivered.

Arousal measure. Tonic EDA is the arousal measure collected during the baseline period prior to administering any stimuli during the Sensory Challenge Protocol. This variable is called baseline skin conductance level and is defined as the average amplitude of 18 sequential 10-s blocks of skin conductance responses recorded over a 3-min period during baseline. During the baseline period the child is asked to sit quietly and no stimuli are presented.

Reactivity measures. Sensory reactivity in each of the six sensory phases is evaluated by four phasic, stimulus-related, EDA variables. The phasic variables include: (1) Orienting response, amplitude of the initial stimulus response (excluding trials in which there was a zero response); (2) Magnitude of response (MAG), amplitude of baseline to peak amplitude averaged across all eight skin conductance responses (including zero for trials in which there was no response) within each sensory domain; (3) Amplitude of response (AMP), the amplitude of baseline to peak amplitude averaged across all skin conductance responses (excluding trials in which there was no response) within each sensory domain; and, (4) non-responding, the percentage of each group that had no response on the first two trials in at least one sensory domain as recommended by Ohman et al. (1989) and Iacono et al. (1999). During the stimulation phase of the experiment, valid responses are defined as peak amplitudes that are greater than $0.02 \mu \mathrm{S}$ and occur between 0.8 and $4.0 \mathrm{~s}$ after stimulus onset.

\section{Parent report measure of sensory-related behaviors}

Short Sensory Profile. Sensory-related behaviors are the parent reported sensory problems reflected by items on the Short Sensory Profile (developed by McIntosh et al. 1999b), a 38-item version of the Sensory Profile (Dunn, 1999). The Short Sensory Profile is comprised of seven subtests, four evaluating parent perceptions of sensory over-responsivity in touch, vision/sound, taste/smell, and movement, one evaluating auditory filtering, one evaluating under-responsivity, called "low energy/weak", and one evaluating sensory seeking. Reliability of the Short Sensory Profile $=0.90$ and discriminant validity is $>95 \%$ (McIntosh et al., 1999a).

\section{PROCEDURES}

ASD and SMD participants attended two sessions. Session one was a Diagnostic Assessment of either (1) ASD, conducted at The Autism and Developmental Research Group at the University of Colorado, Denver, or (2) identification of SMD at The Sensory Therapies And Research (STAR) Center in Greenwood Village, CO. Parent report measures were obtained in Session one. Session two was administration of The Sensory Challenge Protocol Physiological Assessment, conducted at the SPD Foundation in Greenwood Village, CO. Typical controls were screened on a telephone interview to ascertain "normality" based on no prenatal or post-natal complications, good academic achievement and typical socialization as reported by parent. Controls were seen only for Session 2, the Sensory Challenge Protocol Physiological Assessment at the SPD Foundation. Children were compensated for their participation. 


\section{DATA ANALYSES}

Arousal (skin conductance level), reactivity (orienting response, MAG, AMP) and sensory-related behaviors (Short Sensory Profile) were analyzed in a series of repeated measures, mixed model analyses of variance (ANOVAs). The dependent variables were skin conductance level, orienting response, AMP, MAG and Short Sensory Profile subtest raw scores. Group (ASD, SMD, and TYP) and gender were the between condition factors and sensory domain (e.g. visual, auditory, tactile etc.) and trials (1-8) were the repeated measure factors. Bonferroni corrections were applied to adjust for multiple comparisons in post hoc analyses of significant interactions or significant main effects.

The distribution of the electrodermal variables skin conductance level, orienting response, AMP and MAG were evaluated for normality. Neither the arousal nor the reactivity variables were normally distributed (Shapiro-Wilk, $p<0.001$ ). Log transformations were conducted, but significant skewness and kurtosis of the distribution remained. Thus, raw data were used for all analyses because multivariate ANOVAs are robust to departures from normality (Bagiella et al., 2000).

Chi square tests compared non-responding (NR), and mean age across groups.

Correlations and scatterplots were used to examine age trends and relationships among arousal and reactivity variables.

Short Sensory Profile $z$ scores were used to classify participants in each group using a cutpoint of -2 standard deviations below the mean of the standardization sample (McIntosh et al., 1999a) to reflect clinically significant impairment in sensory-related behaviors. Percentages in each group were calculated for Short Sensory Profile subtest impairment.

\section{RESULTS}

\section{PHYSIOLOGY VARIABLES}

Chi square analysis of age revealed no significant differences between groups $\left(\chi^{2}=194.73, p=0.431\right)$, thus age was not included as a covariate in subsequent analyses. Since the gender distribution was different for the typical group compared to the two clinical groups, gender was included as a covariate in the analyses.

\section{Arousal}

Statistical analyses revealed that arousal at baseline (skin conductance level) were significantly lower in the ASD group than either the SMD group or the TYP group (see Figure 1).

There was a significant main effect of group $\left(F_{2,1838}=14.03\right.$; $p<0.001)$ and an interaction between group and gender $\left(F_{2,1838}=11.41 ; p<0.001\right)$. Post hoc pairwise comparisons with Bonferroni adjustments for multiple comparisons showed ASD differed significantly from SMD $(p<0.001)$ and TYP $(p<0.001)$, but SMD did not differ significantly from TYP. Gender differences were as follows: TYP and SMD males had lower baseline arousal than TYP and SMD females; while the reverse was true for ASD (i.e. males had higher baseline arousal than females). However, these gender differences are extremely preliminary since only $7 \%$ of the ASD group and $23 \%$ of the SMD group were females. Thus, possible gender differences need to be explored in another study in which the gender of the participants is more evenly matched.

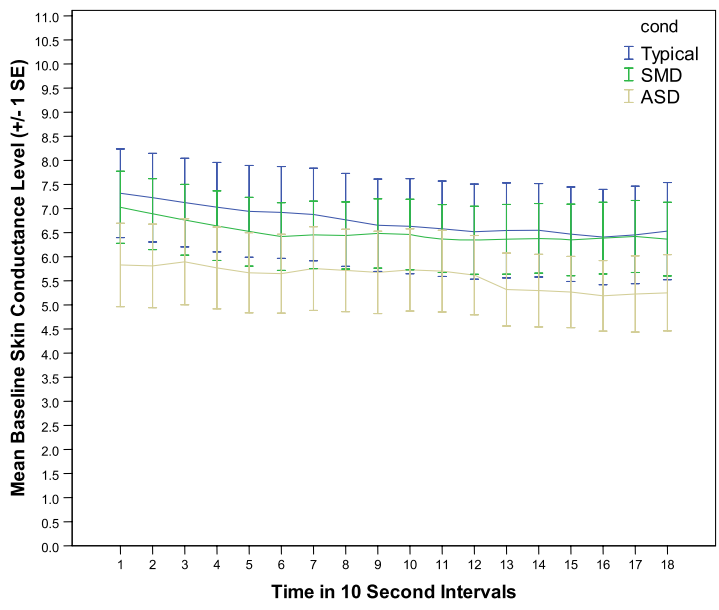

FIGURE 1 |Amplitude of baseline arousal by group.

\section{Reactivity}

There was no significant gender effect or significant interaction between gender and any of the other factors so it was removed from the analyses.

Statistical analyses of three reactivity variables (MAG, AMP and orienting response) demonstrated higher overall reactivity in SMD compared to the ASD and TYP. The ASD group was consistently the lowest across all sensory domains (see Figure 2).

For MAG and AMP there was a significant main effect of domain $\left(F_{5,254}=53.45 ; p<0.001 ; F_{5,409}=43.44, p<0.001\right)$ and a significant interaction between group and domain $\left(F_{10,254}=4.09\right.$, $\left.p<0.001 ; F_{10,409}=2.30, p<0.012\right)$. For orienting response there was a significant main effect of group $\left(F_{2,95}=7.10 ; p<0.001\right)$ and domain $\left(F_{5,247}=41.81 ; p<0.001\right)$. Post hoc pairwise comparisons by domain with Bonferroni adjustments for multiple comparisons revealed that the SMD group had significantly higher values for MAG, AMP and orienting response than the TYP group in 4 of 6 sensory domains (i.e. Tone, Visual, Siren, and Movement; see Figure 2) and compared to the ASD group for MAG and AMP in 5 of 6 sensory domains (i.e. Tone, Visual, Siren, Olfactory and Movement; see Figure 2).

For both MAG and AMP there was also a significant main effect of trial $\left(F_{7,3501}=124.01, p<0.001 ; F_{7,2444}=91.97, p<0.001\right)$ and a significant interaction between group and trial $\left(F_{14,3501}=6.34, p<0.001\right.$, $\left.F_{14,2443}=3.64 ; p<0.001\right)$. A decreasing mean MAG and AMP from trial 1 to trial 8 for all groups across all domains reflects habituation to the stimuli with repeated exposures by all three groups. The interaction between group and trial reflects different rates/amount of change across trials within each group. The SMD group had the greatest amount of change from trial 1 to trial 8 because of the large orienting responses in this group, while the ASD group had smaller orienting responses, thus less range in which they could change and a smaller amount of change from trial 1 to trial 8 .

No significant differences were found for MAG, AMP or orienting response in the olfactory domain among the three groups or in the tactile domain between SMD and TYP. However, the mean response in both olfactory and tactile domains for MAG, AMP and orienting response were higher in SMD than ASD. 

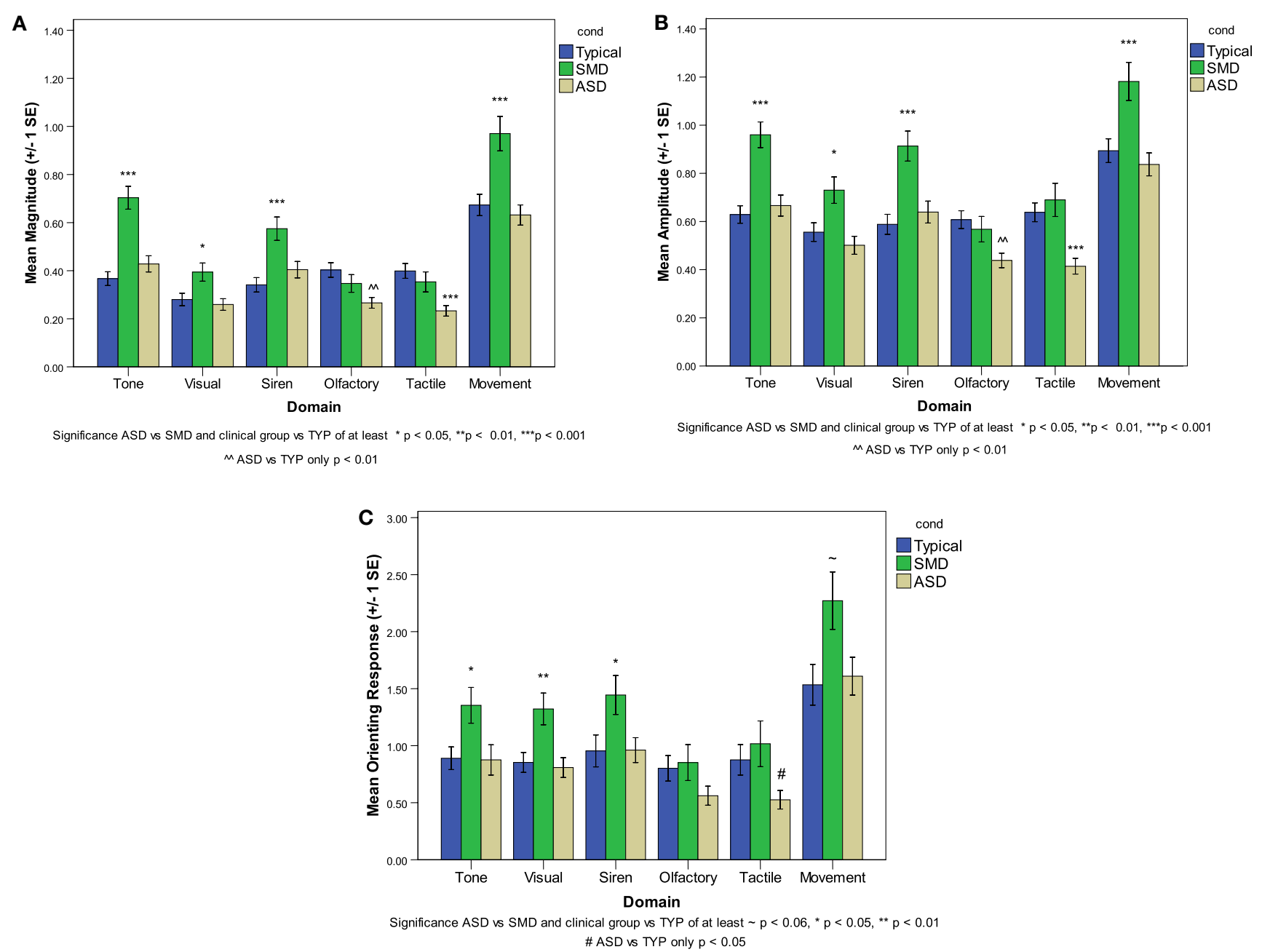

FIGURE 2 | Reactivity variables across groups. (A) Average magnitude, (B) Average amplitude, (C) Average orienting response.

\section{Non-responding}

Nineteen percent of the SMD group was defined as non-responding, whereas $34 \%$ of the ASD group and $27 \%$ of the TYP group were non-responding. There were no significant differences among the three groups $\left(\chi^{2}=1.89 ; p=0.388\right)$.

\section{Association among arousal vs reactivity variables}

The average magnitude (MAG) in each sensory domain was significantly correlated with baseline skin conductance level for both the ASD ( $r=0.439$ to 0.960$)$ and TYP $(r=0.379$ to 0.938$)$ groups. For the SMD group, there were no significant correlations between MAG and baseline skin conductance level.

\section{SENSORY-RELATED BEHAVIORS}

Short Sensory Profile findings are reported in Table 1.

Total Short Sensory Profile scores indicate that $84 \%$ of the ASD group obtained scores below -2 SD as compared to $66 \%$ of the SMD group and none of the TYP group. The ASD and SMD groups both showed significant impairment in auditory filtering, but differed in other sensory-related behaviors. The ASD group had greater
Table 1 | Percentage of groups with clinically significant symptoms.

\begin{tabular}{lll}
\hline & ASD & SMD \\
\hline Total score & $84 \%$ & $66 \%$ \\
Domain & & \\
$\quad$ Auditory filtering & $76 \%$ & $77 \%$ \\
Sensory seeking & $47 \%$ & $61 \%$ \\
Visual/auditory sensitivity & $29 \%$ & $42 \%$ \\
Low energy weak & $71 \%$ & $45 \%$ \\
Tactile sensitivity & $61 \%$ & $42 \%$ \\
Taste/smell sensitivity & $55 \%$ & $32 \%$ \\
Movement sensitivity & $34 \%$ & $23 \%$ \\
\hline
\end{tabular}

impairment in "low energy weak" a measure of proprioceptive and vestibular under-responsivity, tactile sensitivity and taste/smell sensitivity, while the SMD group had greater impairment in sensory seeking and in visual/auditory sensitivity. Neither group showed a high percentage of impairment in movement sensitivity; however the ASD was somewhat higher. 
Statistical analyses of Short Sensory Profile raw scores across the three groups revealed a significant main effect of group $\left(F_{2,536}=176.67, p<0.001\right)$ and domain $\left(F_{6,195}=211.48, p<0.001\right)$ as well as a significant interaction between group and domain $\left(F_{12,195}=7.66, p<0.001\right)$. Both the ASD and the SMD groups were significantly different than the TYP group across all Short Sensory Profile subtests and for the total score $(p<0.001)$. Post hoc pairwise comparisons by subtest between the ASD and SMD groups approached significance in taste/smell sensitivity, with greater impairment reported in the ASD group $(p=0.056)$.

Visual analysis of the data (see Figure 3 ) revealed patterns that were different for ASD and SMD, generating hypotheses for further follow up. The horizontal line represents the cutpoint of -2 standard deviations below the mean of the standardization sample, which was used to reflect areas of dysfunction.

The ASD group had a greater percentage of individuals with clinically significant impairment (i.e. <-2SD) on "low energy weak", a measure of proprioceptive and vestibular under-responivity $\left(\chi^{2}=0.268 ; p<0.028\right)$. Although not significant, the ASD group also had a higher percentage of individuals with taste/smell sensitivity and the SMD group had greater percentage of individuals with clinically significant impairment in sensory seeking. Both groups had clinically significant impairment in tactile sensitivity, but not in movement sensitivity.

\section{RELATIONSHIP AMONG PHYSIOLOGICAL AND SENSORY-RELATED BEHAVIORAL MEASURES}

No significant correlations were found between Short Sensory Profile domains and each of the reactivity variables (MAG, AMP, orienting response).

\section{DISCUSSION}

This study is the first research to compare and contrast the physiological reactions to sensory stimulation and the sensory-related behaviors of children with ASD and children with SMD. Few studies compare physiological and behavioral measures of a clinical population to another group other than typically developing children (Ben-Sasson et al., 2009).

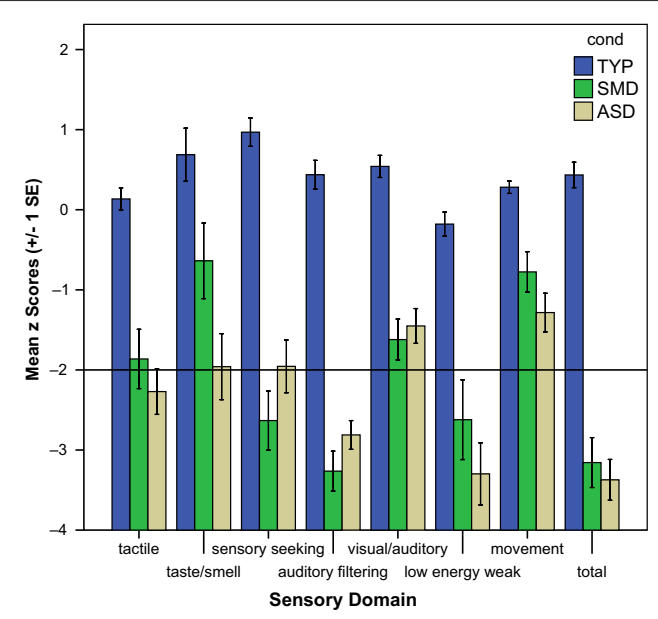

FIGURE 3 | Classification of sensory-related behaviors in ASD and SMD.

\section{PHYSIOLOGICAL MEASUREMENT OF AROUSAL AND REACTIVITY}

Differences in sympathetic nervous system functioning were found between children with ASD compared to children with SMD. As hypothesized, children with ASD had atypical physiological arousal while children with SMD had atypical physiological reactivity. Specifically, the ASD group had significantly lower arousal at baseline than both the SMD and typical controls, and the SMD group had higher reactivity across sensory domains compared to both the ASD and typical controls. Although not reaching statistical significance, the ASD group also had a higher percentage of children who demonstrated non-responding to the first two trials of sensory stimulation in at least before one sensory domain.

These findings confirm previous pilot data using the Sensory Challenge Protocol Space Lab as well as differentiating the ASD and SMD clinical groups. Miller et al. (2001) compared the electrodermal activity (EDA) of children with ASD, fragile X, SMD, Attention deficit/hyperactivity disorder and typical controls. As in the present study, children with ASD were less reactive to all sensory stimuli compared to typically developing children and especially compared to children with SMD, who were also overreactive across all sensory modalities compared to typical controls. Furthermore, this study cross-validates the finding of McIntosh et al. (1999b) of greater physiological reactivity in children with SMD. Stimuli in the Sensory Challenge Protocol are presented passively and do not require an active response on the part of the individual. Therefore, the increased reactivity in children with SMD is assumed to reflect increased perceived significance and impact of the sensory stimuli and not a function of changes in attentional processes. Future studies should explore this hypothesis by including an attention measure.

As in the typical group, both the ASD and the SMD groups displayed progressively lower amplitude responses across the eight trials in each sensory domain, suggestive of habituation. This finding is contrary to a previous pilot study in the Sensory Challenge Protocol Space Lab that showed poor habituation in children with SMD (McIntosh et al., 1999a). Other studies suggest differences in habituation patterns may not exist amongst children with autism, cognitive delay and typical controls (Stevens and Gruzelier, 1984; van Engeland, 1984; van Engeland et al., 1991; Baranek et al., 2007). The current study provides further support that children with clinical disorders demonstrate habituation to repeated sensory stimuli. Thus, it is unlikely that a lack of habituation to sensory stimuli is related to the atypical sensory-related behaviors of these children with ASD or SMD.

Rather, differences in rates of non-responding (i.e. not orienting to novel stimuli) in young children may correspond to the atypical sensory-related behaviors reported in ASD. Deficits in orienting responses to social and non-social sensory stimuli have been found in young children with ASD (Dawson et al., 1998; Baranek et al., 2007). Although not significant, the current study also found the highest percentage of non-responding in the ASD group. The lack of significance in the current study may be influenced by the older age (5-13 years) of the current ASD sample compared to the previous studies ( 5 months to 6 years) that found significantly less orienting (Dawson et al., 1998; Baranek et al., 2007). Other studies have also reported children with autism not responding to the first sensory stimulus (van Engeland, 1984; Hirstein et al., 2001; Schoen 
et al., 2008b). Consequently, in young children a lack of orienting may be an early indicator of ASD and may also differentiate young children with autism from those with SMD. Additional studies are needed to determine if lack of orienting is a consistent finding in children with ASD.

A strong association was found between baseline arousal and reactivity in ASD and typical controls, but not in SMD children; children in the ASD and TYP groups who had higher arousal, tended to have higher reactivity, while those with lower arousal tended to have lower reactivity. This finding is consistent with previous research in children with ASD (Schoen et al., 2008a) that demonstrated a similar relationship between arousal and reactivity measures. However, unlike Schoen et al. (2008a), two separate patterns of arousal and reactivity, (i.e. high, high and low, low) were not apparent in the present study. Rather, the children with ASD in this study seemed to reflect only physiologic hypo-arousal, especially when compared to children with SMD or typical controls. On the other hand, children with SMD had consistently high reactivity regardless of whether their arousal was high or low at baseline. This corresponds to clinical observations of children with SMD, in that those who are over-responsive to sensory input often become over-aroused (Lane, 2002). This suggests that high physiological reactivity in children with SMD may be related to behavioral observations of over-arousal even though the underlying physiological mechanisms of arousal and reactivity are different.

The two atypical patterns of EDA found in this study (i.e. hypoarousal in ASD and heightened reactivity in SMD compared to typical controls) are similar to reports in the schizophrenia literature. In schizophrenia atypical patterns of EDA predict functional outcomes (Schell et al., 2005). Schell et al. (2005) suggest that the relationship between EDA and good outcomes can be understood by Hebb's (1949, 1955) classic inverted-U relationship between arousal and performance. This theoretical perspective depicts optimal performance as dependent on both arousal level and perceived stimulus intensity and that both ends of the continuum represent abnormality. It is possible that our sample of children with SMD resemble individuals with schizophrenia who have atypically high EDA. Schell et al. (2005) propose that these individuals have impaired information processing resulting in misinterpretation of stimuli and a reduction in one's ability to discriminate between relevant and irrelevant information. Similarly, our sample of children with ASD resembles individuals with schizophrenia who have extremely low levels of arousal. These individuals may have a diminished capacity to allocate attentional resources to process environmental stimuli (Schell et al., 2005).

Practitioners working with children with ASD or SMD often note that over-reactivity can lead to over-arousal, anxiety, attention problems, behavioral disorganization and poor adaptive environmental interactions (Lane, 2002; Miller et al., 2007b). In addition, under-reactivity can co-occur with under-arousal, decreased awareness of environmental stimuli and seeming lack of inner drive to initiate socialization and exploration (Miller et al., 2007b). Although arousal and sensory reactivity are physiologically different, clinically children often present with both behavioral over-responsivity to sensory input and concomitant signs of over-arousal, or behavioral under-responsivity and under-arousal, which impair their ability to function successfully in daily life activities (Lane, 2002).
Behaviors seen in children with SMD often resemble some behaviors of children with anxiety disorders or Attention Deficit Hyperactivity Disorder (ADHD). Of the thirty-one participants with SMD, one had a clinical diagnosis of Generalized Anxiety Disorder and four had ADHD. Since all other SMD and ASD participants were not screened for an anxiety or ADHD diagnosis, it is unknown the extent to which co-morbidity may have impacted these findings. In addition, the psychophysiology literature suggests patterns of arousal and reactivity in children with anxiety (Rabavilas, 1989; Birket-Smith et al., 1993; Jensen et al., 1996) or ADHD (Shibagaki et al., 1993; Zahn and Kruesi, 1993; O'Connell et al., 2004; Lawrence et al., 2005) which should be differentiated from children with SMD in future studies.

\section{MEASUREMENT OF SENSORY-RELATED BEHAVIORS}

Both ASD and SMD groups had significantly more sensory-related behavioral symptoms than typically developing controls. In addition, both similarities and differences were observed between children with ASD compared to children with SMD. Similarities between the two clinical groups included impairment in auditory filtering and tactile sensitivity, with few symptoms of movement sensitivity. However, the SMD group was characterized by higher sensory seeking behavior, while the ASD group had a higher percentage of low energy weak scores, possibly indicative of sensory under-responsivity. The ASD group also had more impairment in taste/smell sensitivity, similar to previous reports (Ermer and Dunn, 1998; Miller et al., 2001; Lane, 2002; Rogers et al., 2003).

Previous studies comparing sensory-related behaviors of children with autism to typically developing children or to children with other developmental delays, have found that the ASD group demonstrate greater Sensory Under-Responsivity (as reviewed in Ben-Sasson et al., 2009). In fact, Sensory Under-Responsivity (also known as hypo-responsiveness) in ASD is reported to be the most common atypical sensory-related behavioral problem in ASD relative to the other sensory modulation subtypes (Rogers and Ozonoff, 2005; Adamson et al., 2006; Baranek et al., 2006; Ben-Sasson et al., 2007). One of the limitations of the Short Sensory Profile scale is that the sensory seeking subtest is labeled under-responsivity/ sensory seeking; however, in more recent conceptual models, the "low energy/weak" subtest has been shown to be a better indicator of sensory under-responsivity in the vestibular and proprioceptive domains (Miller et al., 2007b). Using "low energy/weak" to reflect sensory under-responsivity, the ASD group in this study had a significantly higher frequency of sensory under-responsivity and a lower frequency of sensory seeking behaviors than the SMD group.

Some research suggests that incidence of taste/smell sensitivity is greater in children with ASD (Ermer and Dunn, 1998; Miller et al., 2001; Rogers et al., 2003). The current study also showed a greater percentage of clinically significant symptoms of taste/smell sensitivity in children with ASD compared to SMD. Furthermore, research suggests that feeding problems in some children with ASD may be the result of over-responsivity to food textures, tastes or smells (Schreck and Williams, 2006; Martin et al., 2008). The present study found $57 \%$ of children with ASD had symptoms of taste/smell sensitivity. Consistent with this finding, a recent study identified $52 \%$ of their sample had symptoms of taste/smell sensitivity (Lane et al., 2009). That study suggested sensory subtypes in ASD could be 
discriminated from each other by atypical versus typical taste/smell sensitivity (Lane, 2009, submitted). Further examination of sensory subtypes and the role of taste/smell sensitivity are warranted based on the findings of the present study.

\section{RELATIONSHIP BETWEEN PHYSIOLOGICAL MEASURES AND MEASURES OF SENSORY-RELATED BEHAVIORS}

This study found no association between behavioral and physiologic measures of sensory processing for either the ASD or SMD groups. Similarly, a recent review of the literature on sensory symptoms in children with ASD highlighted the discrepancy between parent report measures and physiological findings of sensory functioning (Rogers and Ozonoff, 2005). Several explanations for this discrepancy are plausible. First, the participants within the ASD and SMD groups may not be homogeneous. Since both clinical groups consisted of individuals with Sensory OverResponsivity, Sensory Under-Responsivity and Sensory Seeking/ Craving it is possible that these behavioral subtypes exhibit different physiological activity (i.e. baseline arousal and reactivity to stimuli). While the specific patterns of physiologic activity by subtype are not known, the lack of a larger sample size in this study did not allow for exploration of differences by subtype. However, it is possible that extremes of high or low physiologic activity may characterize a specific subtype. This heterogeneity, and potential difference in physiological patterns in each group, may have contributed to the lack of relationship between the physiological data and the parent report measures. Future studies should examine differences in the physiology of individuals based on behavioral subtypes (i.e. Sensory Over-Responsivity, Sensory Under-Responsivity and Sensory Seeking/Craving). Second, parent questionnaires are limited to the constructs identified by items in the scale. On the Short Sensory Profile in particular, Sensory-Under-Responsivity and Sensory-Seeking/Craving items are under-represented. Third, parent questionnaires are reported to be less than perfectly correlated with clinician observations of sensory-related behaviors (Ben-Sasson et al., 2009). While parent questionnaires have the advantage of sampling a large number of behaviors across place and time from an observer highly familiar with the child, caution is raised because the responses can be

\section{REFERENCES}

Adamson, A., O’Hare, A., and Graham, C. (2006). Impairments in sensory modulation in children with autism spectrum disorders. Br.J. Occup. Ther. 69, 357-364

Adrien, J. L., Ornitz, E. M., Barthelemy, C., Sauvage, D., and Lelord, G. (1987). The presence or absence of certain behaviors associated with infantile autism in severely retarded autistic and nonautistic retarded children and very young normal children. J.Autism Dev. Disord. 17, 407-416.

Ahn, R. R., Miller, L. J., Milberger, S., and McIntosh, D. N. (2004). Prevalence of parents' perceptions of sensory processing disorders among kindergarten children. Am. J. Occup. Ther. 58, 287-302.
Ashburner, J., Ziviani, J., and Rodger, S. (2008). Sensory processing and classroom emotional, behavioral, and educational outcomes in children with autism spectrum disorder. Am. J. Occup. Ther. 62, 564-573.

Bagiella, E., Sloan, R. P., and Heitjan, D. F. (2000). Mixed-effects models in psychophysiology. Psychophysiology 37, 13-20.

Baker, A. E., Lane, A., Angley, M. T., and Young, R. L. (2008). The relationship between sensory processing patterns and behavioural responsiveness in autistic disorder: a pilot study. J. Autism Dev. Disord. 38, 867-875.

Bar-Shalita, T., Goldstand, S., HahnMarkowitz, J., and Parush, S. (2005). Typical children's responsivity patterns of the tactile and vestibular

influenced by the symptoms parents know are associated with their child's diagnosis (Dahlgren and Gillberg, 1989; Rogers et al., 2003). Some of the literature highlights the importance of developing direct observation measures to identify sensory modulation disorder (Baranek et al., 2007; Schoen et al., 2008a). Collectively these studies suggest that a performance measure of sensory-related behaviors should be used in future studies to cross validate information acquired through parent report (BenSasson et al., 2009).

\section{CONCLUSION}

In summary, this study confirmed previous literature differentiating children with ASD and children with SMD from typically developing children on both behavioral and physiological measures. In addition, the two clinical groups differed from each other. Children with ASD had lower baseline arousal and lower reactivity when exposed to a laboratory paradigm of sensory challenges. Children with SMD had high reactivity across all sensory domains especially to the first trial of each sensory stimulus. Both clinical groups had significantly more atypical sensory-related behaviors than typically developing children and differed from each other in three ways; (1) the ASD group had more symptoms of taste smell over-responsivity; (2) the SMD group displayed more sensory seeking behaviors and (3) the ASD group displayed greater sensory under-responsivity in the vestibular and proprioceptive domains. Further study is warranted with lower functioning children with ASD to determine whether the results of this study can be extended across the spectrum of children with autism. Differentiating the physiological and sensory-related behaviors of clinical groups has important implications for understanding each clinical disorder, improving diagnostic accuracy and planning appropriate treatment.

\section{ACKNOWLEDGMENTS}

We wish to thank the parents and children who so generously volunteered their time to participate in this study. Funding for this work was provided by the Wallace Research Foundation and Cure Autism Now. The support of staff at the Sensory Therapies And Research Center and the Sensory Processing Disorder (SPD) Foundation is also gratefully acknowledged.

systems. Am. J. Occup. Ther. 59 148-156.

Bar-Shalita, T., Vatine, J., and Parush, S. (2008). Sensory modulation disorder: a risk factor for participation in daily life activities. Dev. Med. Child Neurol. 50, 932-937.

Baranek, G. T. (1999). Autism during infancy: a retrospective video analysis of sensory-motor and social behaviors at 9-12 months of age. J. Autism Dev. Disord. 29, 213-224.

Baranek, G. T. (2002). Efficacy of sensory and motor interventions for children with autism. J. Autism Dev. Disord. 32, 397-422.

Baranek, G. T., Boyd, B. A., Poe, M. D., David, F. J., and Watson, L. R. (2007). Hyperresponsive sensory patterns in young children with autism, developmental delay, and typical development.Am. J.Ment. Retard. 112, 233-245.

Baranek, G. T., David, F. J., Poe, M. D., Stone, W. L., and Watson, L. R. (2006). Sensory experiences questionnaire: discriminating sensory features in young children with autism, developmental delays, and typical development. J. Child Psychol. Psychiatry 47, 591-601.

Barry, R. J., and James, A. L. (1988). Coding of stimulus parameters in autistic, retarded, and normal children: evidence for a two-factor theory of autism. Int. J. Psychophysiol. 6, 139-149.

Ben-Sasson, A., Carter, A. S., and BriggsGowan, M. J. (2009). Sensory overresponsivity in elementary school: 
prevalence and social-emotional correlates. J. Abnorm. Child. Psychol. 5, 705-716.

Ben-Sasson, A., Cermak, S. A., Orsmond, G. I., Tager-Flusberg, H., Carter, A. S., Kadlec, M. B., and Dunn, W. (2007). Extreme sensory modulation behaviors in toddlers with autism spectrum disorders. Am. J. Occup. Ther. 61, 584-592.

Berument, S. K., Rutter, M., Lord, C., Pickles, A., and Bailey, A. (1999). Autism screening questionnaire: diagnostic validity. Br. J. Psychiatry 175, 444-451.

Birket-Smith, M., Hasle, N., and Jensen, H. H. (1993). Electrodermal activity in anxiety disorders. Acta Psychiatr. Scand. 88, 350-355.

Boucsein, W. (1992). Electrodermal Activity. New York, Plenum Press.

Cohn,E., Miller,L.J., and Tickle-Degnen, L. (2000). Parental hopes for therapy outcomes: children with sensory modulation disorders. Am. J. Occup. Ther. 54, 36-43.

Dahlgren, S. O., and Gillberg, C. (1989). Symptoms in the first two years of life: a preliminary population study of infantile autism. Eur. Arch. Psychiatry Neurol. Sci. 238, 169-174.

Dawson, G., and Lewy, A. (1989). Arousal, attention, and the socioemotional impairments of individuals with autism. In Autism: Nature, Diagnosis, and Treatment, G. Dawson, ed. (New York, Guilford), pp. 49-74.

Dawson, G., Meltzoff, A. N., Osterling, J., Rinaldi, J., and Brown, E. (1998). Children with autism fail to orient to naturally occurring social stimuli. J. Autism Dev. Disord. 28, 479-485.

Dawson, M. E., Schell, A. M., and Filion, D. L. (2000). The electrodermal system. In Handbook of Psychophysiology, J. Cacioppo, L. G. Tassinary, and G. G. Berntson, eds (New York, Cambridge University Press), pp. 200-223.

DesLauriers, A. M., and Carlson, C. F. (1969). Your child is asleep: early infantile autism. Homewood, IL, The Dorsey Press.

Diagnostic Manual for Infancy and Early Childhood (2005). Mental Health, Developmental, Regulatory-Sensory Processing and Language Disorders and Learning Challenges (icdl-dmic). Bethesda, MD, Interdisciplinary Council on Developmental and Learning Disorders (ICDL).

Dunn, W. (1997a). The impact of sensory processing abilities on the daily lives of young children and their families: a conceptual model. Infants Young Child. 9, 23-35.

Dunn, W. (1997b). The sensory profile: a discriminating measure of sensory processing in daily life. Sens.
Integration Spec. Interest Sect. Q. 20, $1-3$.

Dunn, W. (1999). The Sensory Profile: Examiner's Manual. San Antonio, TX, The Psychological Corporation.

Dunn, W. (2001). The sensations of everyday life: empirical, theoretical, and pragmatic considerations. Am. J. Occup. Ther. 55, 608-620.

Edelberg, R. (1973). Mechanisms of electrodermal adaptations for locomotion, manipulation, or defense. Prog. Physiol. Psychol. 5, 155-209.

Ermer, J., and Dunn, W. (1998). The sensory profile: a discriminate analysis of children with and without disabilities. Am. J. Occup. Ther. 52, 283-290.

Hagerman, R. J., Miller, L. J., McGrathClarke, J., Riley, K., Goldson, E., Harris, S. W., Simon, J., Church, K., Bonnell, J., Oqnibene, T. C., and McIntosh, D. N. (2002). Influence of stimulants on electrodermal studies in Fragile X syndrome. Microsc. Res. Tech. 57, 168-173.

Hebb, D. O. (1949). Organization of Behavior. New York, Wiley.

Hebb, D. O. (1955). Drives and the CNS (conceptual nervous system). Psychol. Rev. 62, 243-254.

Hilton, C., Graver, K., and LaVesser, P. (2007). Relationship between social competence and sensory processing in children with high functioning autism spectrum disorders. Res. Autism Spectr. Disord. 1, 164-173.

Hirstein,W.,Iversen,P., andRamachandran, V.S. (2001). Autonomic responses of autistic children to people and objects. Proc. R. Soc. Lond., B, Biol. Sci. 268, 1883-1888.

Hugdahl, K. (1995). Psychophysiology: The Mind-Body Perspective. Cambridge, MA, Harvard University Press.

Hutt, C., and Hutt, S. J. (1964). Arousal and childhood autism. Nature 204, 908-909.

Iacono, W. G., Ficker, J. W., and Beiser, M. (1999). Electrodermal activation in first-episode psychotic patients and their first-degree relatives. Psychiatry Res. 88, 25-39.

Iarocci, G., and McDonald, J. (2006). Sensory integration and the perceptual experience of persons with autism. J. Autism Dev. Disord. 36, 77-90.

James, A. L., and Barry, R. J. (1984). Cardiovascular and electrodermal responses to simple stimuli in autistic, retarded and normal children. Int. J. Psychophysiol. 1, 179-193.

Jensen, H. H., Hasle, N., and BirketSmith, M. (1996). Electrodermal lability in anxiety disorders. Scand. J. Psychol. 37, 103-108.

Kern, J. K., Garver, C. R., Carmody, T., Andrews, A.A., Mehta, J.A., and Trivedi,
M. H. (2008). Examining sensory modulation in individuals with autism as compared to community controls. Res. Autism Spectr. Disord. 2, 85-94.

Kern, J. K., Garver, C. R., Carmody, T. Andrews, A. A., Trivedi, M. H., and Mehta, J. A. (2007). Examining sensory quadrants in autism. Res. Autism Spectr. Disord. 1, 185-193.

Kern, J. K., Trivedi, M. H., Garver, C. B., Grannemann, B. D., Andres, A. A. Salva, J. S., Johnson, D. G., Mehta, J. A. and Schroeder, J.L. (2006). The pattern of sensory processing abnormalities in autism. Autism 10, 480-494.

Kientz, M. A., and Dunn, W. (1997). A comparison of the performance of children with and without autism on the sensory profile. Am. J. Occup. Ther. 51, 530-537.

Kinnealey, M., Oliver, B., and Wilbarger, P. (1995). A phenomenological study of sensory defensiveness in adults. Am. J. Occup. Ther. 49, 444-451.

Lane, A. E., Young, R. L., Baker, A. E. and Angley, M. T. (2009). Sensory processing subtypes in autism: Association with adaptive behavior. J. Autism. Dev. Disord. doi: 10.1007/s10803-009-0840-2.

Lane, S. J. (2002). Sensory modulation. In Sensory Integration: Theory and Practice, A. C. Bundy, S. J. Lane, and E. A. Murray, eds (Philadelphia, PA, F.A. Davis Company), pp. 101-122.

Lawrence, C. A., Barry, R. J., Clarke, A R., Johnstone, S. J., McCarthy, R., Selokowitz, M., and Broyd, S. J. (2005). Methylphenidateeffectsinattention deficit/hyperactivity disorder: electrodermal and erp measures during a continuous performance task. Psychopharmacology (Berl.) 183, 81-91.

Lee, G. P., Arena, J. G., Meador, K. J., Smith, J. R., Loring, D. W., and Flanigin, H. F. (1988). Change in autonomic responsiveness following bilateral amygdalotomy in humans. Neuropsychiatry Neuropsychol. Behav. Neurol. 1, 119-129.

Leekam, S. R., Libby, S. J., Wing, L., and Gould, J. (2007). Describing the sensory abnormalities of children and adults with autism. J. Autism Dev. Disord. 37, 894-910.

Liss, M., Saulnier, C., Fein, D., and Kinsbourne, M. (2006). Sensory and attention abnormalities in autistic spectrum disorders. Autism 10 155-172.

Lord, C., Rutter, M., DiLavore, P. C., and Risi, S. (1999). Autism diagnostic observation schedule (ADOS). Los Angeles, CA, Western Psychological Services.

Mangeot, S. D., Miller, L. J., McIntosh, D. N., McGrathClarke, J., Simon, J., Hagerman, R.
J., and Goldson, E. (2001). Sensory modulation dysfunction in children with attention-deficit-hyperactivity disorder. Dev. Med. Child Neurol. 43, 399-406.

Mangina, C. A., and BeuzeronMangina, J. H. (1996). Direct electrical stimulation of specific human brain structures and bilateral electrodermal activity. Int. J. Psychophysiol. 22, 1-8.

Martin, Y., Young, R. Y., and Robson, D. C. (2008). Feeding and eating bahaviors in children with autism and typically developing children. J. Autism Dev. Disord. 38, 1878-1887.

Martinez-Selva, J. M., GarciaSanchez, F. A., and Florit, R. (1995). Electrodermal orienting activity in children with down syndrome. Am. J. Ment. Retard. 100, 51-58.

McIntosh, D. N., Miller, L. J., Shyu, V., and Dunn, W. (1999a). Overview of the short sensory profile (SSP). In The Sensory Profile: Examiner's Manual, W. Dunn, ed. (San Antonio, TX, The Psychological Corporation), pp. 59-73.

McIntosh, D. N., Miller, L. J., Shyu, V., and Hagerman, R. (1999b). Sensorymodulation disruption, electrodermal responses, and functional behaviors. Dev. Med. Child Neurol. 41, 608-615.

Miller, L., Anzalone, M., Lane, S., Cermak, S., and Osten, E. (2007a). Concept evolution in sensory integration: a proposed nosology for diagnosis. Am. J. Occup. Ther. 61, 135-140.

Miller, L. J., Coll, J. R., and Schoen, S. A. (2007b). A randomized controlled pilot study of the effectiveness of occupational therapy for children with sensory modulation disorder. Am. J. Occup. Ther. 61, 228-238.

Miller, L., McIntosh, D., McGrath, J., Shyu, V., Lampe, M., Taylor, A. K., Tassone, F., Neitsel, K., Stackhouse, T., and Hagerman, R. (1999). Electrodemal responses to sensory stimuli in individuals with Fragile X syndrome: a preliminary report. Am. J. Med. Genet. 83, 268-279.

Miller,L. J., Reisman, J.E., McIntosh, D. N. and Simon, J. (2001). An ecological model of sensory modulation: performance of children with Fragile X syndrome, autistic disorder, attention-deficit/hyperactivity disorder, and sensory modulation dysfunction. In Understanding the Nature of Sensory Integration With Diverse Populations, S. S. Roley, E. I. Blanche, and R. C. Schaaf, eds (San Antonio, Therapy Skill Builders), pp. 57-88.

Minshew, N. J., and Hobson, J. A. (2008). Sensory sensitivities and performance on sensory perceptual tasks in 
high-functioning individuals with autism. J. Autism Dev. Disord. 38, 1485-1498.

O’Connell, R. G., Bellgrove, M. A., Dockree, P. M., and Robertson, I. H. (2004). Reduced electrodermal responses to errors predicts poor sustained attention performance in attention deficit hyperactivity disorder. Neuroreport 15, 2535-2538.

Ohman, A., Ohlund, L. S., Alm, T., Wieselgren, I. M., and Ost, L. G. (1989). Electrodermal nonresponding, premorbid adjustment, and symptomatology as predictors of long-term social functioning in schizophrenics. J. Abnorm. Psychol. 98, 426-435.

Palkovitz, R. J., and Wiesenfeld, A. R. (1980). Differential autonomic responses of autistic and normal children. J. Autism Dev. Disord. 10, 347-360.

Pfeiffer, B., Kinnealey, M., Reed, C., and Herzberg, G. (2005). Sensory modulation and affective disorders in children and adolescents with asperger's disorder. Am. J. Occup. Ther. 59, 335-345.

Rabavilas,A.D.(1989).Clinical significance of the electrodermal habituation rate in anxiety disorders. Neuropsychobiology $22,68-71$.

Reynolds, S., and Lane, S. J. (2008). Diagnostic validity of sensory overresponsivity: a review of the literature and case reports. J. Autism Dev. Disord. 38, 516-529.

Rimland, B. (1964). Infantile Autism. New York, Appleton-Century-Crofts.

Rogers, S. J., Hepburn, S., and Wehner, E. (2003). Parent reports of sensory symptoms in toddlers with autism and those with other developmental disorders. J. Autism Dev. Disord. 33, 631-642.

Rogers, S. J., and Ozonoff, S. (2005). Annotation: what do we know about sensory dysfunction in autism? A critical review of the empirical evidence.J. Child Psychol. Psychiatry 46, 1255-1268.

Schaaf, R. C., Miller, L. J., Sewell, D., and O'Keefe, S. (2003). Children with disturbances in sensory processing: a pilot study examining the role of the parasympathetic nervous system. Am. J. Occup. Ther. 57, 442-449.

Schell, A. M., Dawson, M. E., Rissling, A., Ventura, J., and Subotnik, K. L. (2005). Electrodermal predictors of functional outcome and negative symptoms in schizophrenia. Psychophysiology 42, 483-492.

Schoen, S. A., Miller, L. J., BrettGreen, B., and Hepburn, S. L. (2008a). Psychophysiology of children with autism spectrum disorder. Res. Autism Spectr. Disord. 2, 417-429.

Schoen, S. A., Miller, L. J., Brett-Green, B., Reynolds, S., and Lane, S. J. (2008b). Arousal and reactivity in children with sensory processing disorder and autism spectrum disorder. Psychophysiology 45, S102.

Schreck, K. A., and Williams, A. (2006). Food preferences and factors influencing food selectivity for children with autism spectrum disorders. Res. Dev. Disabil. 27, 353-363.

Sequeira, H., Hot, P., Silvert, L., and Delplanque, S. (2009). Electrical autonomic correlates of emotion. Int. J. Psychophysiol. 71, 50-56.
Shibagaki, M., Yamanaka, T., and Furuya, T. (1993). Attention state in electrodermal activity during auditory stimulation of children with attention-deficit hyperactivity disorder. Percept. Mot. Skills 77, 331-338.

Smith, A. M., Roux, S., Naidoo, N. T., and Venter, D. J. (2005). Food choices of tactile defensive children. Nutrition 21, 14-19.

Stevens, S., and Gruzelier, J. (1984) Electrodermal activity to auditory stimuli in autistic, retarded, and normal children. J. Autism Dev. Disord. 14, 245-260.

Talay-Ongan, A., and Wood, K. (2000). Unusual sensory sensitivities in autism: a possible crossroads. Int J. Disabil. Dev. Ed. 47, 201-212.

Tomchek, S. D., and Dunn, W. (2007). Sensory processing in children with and without autism: a comparative study using the short sensory profile. Am. J. Occup. Ther. 61, 190-200.

van Engeland, H. (1984). The electrodermal orienting response to auditive stimuli in autistic children, normal children, mentally retarded children, and child psychiatric patients. J. Autism Dev. Disord. 14, 261-279.

van Engeland, H., Roelofs, J. W., Verbaten, M. N., and Slangen, J. L. (1991). Abnormal electrodermal reactivity to novel visual stimuli in autistic children. Psychiatry Res. 38, 27-38.

Watling, R. L., Deitz, J., and White, O. (2001). Comparison of sensory profile scores of young children with and without autism spectrum disorders. Am. J. Occup. Ther. 55, 416-423.
World Health Organization. (2001). International Classification of Functioning, Disability and Health. Geneva, Switzerland.

Zahn, T. P., and Kruesi, M. P. (1993). Autonomic activity in boys with disruptive behavior disorders. Psychophysiology 30, 605-614.

Zero To Three. (2005). Diagnostic Classification of Mental Health and Developmental Disorders of Infancy and Early Childhood: Revised Edition (dc:0-3r). Washington, DC, ZERO TO THREE Press.

Conflict of Interest Statement: The authors declare that the research was conducted in the absence of any commercial or financial relationships that could be construed as a potential conflict of interest.

Received: 30 March 2009; paper pending published:27 July 2009; accepted: 13 October 2009; published online: 03 November 2009. Citation: Schoen SA, Miller LJ, BrettGreen BA and Nielsen DM (2009) Physiological and behavioral differences in sensory processing: a comparison of children with Autism Spectrum Disorder and Sensory Modulation Disorder. Front. Integr. Neurosci. 3:29. doi: 10.3389/neuro.07.029.2009

Copyright (C) 2009 Schoen, Miller, BrettGreen and Nielsen. This is an open-access article subject to an exclusive license agreement between the authors and the Frontiers Research Foundation, which permits unrestricted use, distribution, and reproduction in any medium, provided the original authors and source are credited. 